\title{
COMBINATION OF PLATELET-RICH PLASMA AND HYALURONIC ACID IN THE TREATMENT OF MODERATE AND SEVERE OSTEOARTHRITIS OF THE KNEE JOINT
}

\author{
M. Razaq ${ }^{1}$, A. Qasim ${ }^{2}$, Th. Assim ${ }^{3}$, R. Ramzi ${ }^{3}$ \\ ${ }^{1}$ Orthopedic Department, College of Medicine, University of Mosul - Mosul, Iraq \\ ${ }^{2}$ Department of Orthopedic Surgery, Aljamhoori Teaching Hospital, Mosul Health Directorate, \\ Ministry of Health/Environment - Mosul, Iraq \\ ${ }^{3}$ Department of Cardiothoracic Surgery, Aljamhoori Teaching Hospital, Mosul Health Directorate, \\ Ministry of Health/Environment - Mosul, Iraq
}

\begin{abstract}
The study aimed to determine the effectiveness of monthly intra-articular injection of a combination of platelet-rich plasma (PRP) and hyaluronic acid in the improvement of outcome of knee OA. It was conducted as an open clinical trial on a convenient sample of 17 patients with unilateral or bilateral symptomatic knee $O A$ in rheumatology and orthopedic clinics at Al-Jumhoori Teaching Hospital in Mosul, Iraq from 20 January to 30 December, 2018. The patients received an intra-articular injection of a combination of PRP and hyaluronic acid (HA) monthly for three months. The outcome assessment was carried out 4 weeks after the last injection using the WOMAC index. The results revealed that WOMAC index subscales was reduced significantly ( $p$ < 0.001) after treatment by 3.88 in pain (95\% Cl: 3.18-4.58); 1.29 in stiffness (95\% Cl: 0.85-1.73); 9.29 in physical function (95\% Cl: 6.70-11.88); and 14.47 in total score (95\% Cl: 11.20-17.73). The intervention effect sizes were as follows: 1.23 in pain; 1.49 in stiffness; 1.11 in physical function, and 1.20 in total WOMAC score. Overall, the effect size of the combination of PRP and HA intra-articular injection is classified as large effect size (>0.8) in improving the outcome of knee OA. A combination of PRP and HA has a large synergistic effect size in improving the outcome of moderate and severe $O A$ of the knee.
\end{abstract}

Key words: Knee osteoarthritis, intra-articular injection, PRP, HA

\section{INTRODUCTION}

Osteoarthritis $(O A)$ is a degenerative disease, manifested as arthralgia, stiffness, and deformities of the affected joint, and is rated as top disabling illness among adults with serious impairment of function [1]. It commonly affects the knee joint because of their size and site, due to the knee is the largest joint in the body and involved in most of the daily activities [2]. These repetitive micro and macro trauma since the early years of life expose the knees to maximum stress and strain [2]. The inherent low healing potential of the chondral disease makes OA management a challenging task [2, 3]. Two therapeutic approaches have exhibited a potential efficacy in the restoration of the normal articular homeostasis. The first one is the use of freshly prepared injection of autologous platelet-riches plasma (PRP), and the results revealed promote proliferation and differentiation of the synovial cell, and also promote recovery of cartilage morphology [4]. The other approach is the intra-articular injection of hyaluronic acid $(\mathrm{HA})$ to improve the viscoelastic and lubricant capabilities of the synovial fluid in joints. HA was found to relieve pain and improve the quality of life of patients with
OA [5]. The synergistic effect of combining PRP and $\mathrm{HA}$ injection in the relieving symptom and improving the physical function of knee $O A$ is not investigated [6]. Görmeli et al found that single intra-articular injections of PRP or HA did not achieve the ideal therapeutic effect for patients with osteoarthritis [7]. And maybe the knowledge of the effectiveness of concomitant serial injection in treating knee $\mathrm{OH}$ is lacking. These gaps in knowledge prompt us to conduct this trial.

The study aimed to determine the effectiveness of monthly intra-articular injection of a combination between PRP and hyaluronic acid for improvement of the outcome of the knee OA.

\section{MEthOdS}

\section{Study design and setting}

The study was conducted as an open clinical trial. A convenient sample of compliant patients with unilateral or bilateral symptomatic knee OA (diagnosis based on clinical and radiological criteria) who attended to rheumatology and orthopedic clinics at Al-Jumhoori Teaching Hospital, Mosul, Iraq. The 
study conducted from 20 January 2018 to 30 December 2018.

\section{Inclusion criteria}

1. The participants informed about the benefits and possible adverse effects of intervention in a written form.

2. Patients with knee OA more than one-year duration.

\section{Exclusion criteria}

1. The patients with unstable medical conditions

2. Coagulation disorders

3. Knee surgery within 3 months

4. Previous infection of the knee joint

5. Pregnant and breastfeeding mothers

\section{Data collection}

The researchers interviewed the patients, and collect baseline demographic and clinical data.

\section{PRP/HA preparation/injection procedure}

Then patients received their first intra-articular injection of a combination of PRP and HA. They were scheduled to receive two additional injections separated by a 4-week interval. The outcome assessment data was carried out 4 weeks after the last injection for each process of injection. The PRP injection was prepared by taking fresh blood at each session. $5 \mathrm{ml}$ of the patient's autologous blood was aspirated by venipuncture (for each patient). PRP preparation standardized recommendation was adhered to achieve maximum platelet concentration. The aspirated blood was injected into a tube containing anticoagulant, separation factor, and $2 \mathrm{ml}$ of non-cross-linked hyaluronic acid. The blood was centrifuged into 2 layers using $3600 \mathrm{cycle} / \mathrm{min}$ for 16 minutes 2 times. The supernatant $2.5 \mathrm{ml}$ plasma rich in platelet and $2 \mathrm{ml}$ hyaluronic acid was isolated and injected into the patient's knee under a sterile environment using the superolateral approach. The site of injection was anesthetized by spraying the area by Ethinyl chloride. We did not utilize the tubes with the PRP chamber because it was unavailable.

\section{Indexing of patient-reported outcome}

1. The baseline and outcome OA assessment was performed using the Arabic translation of the Western Ontario and McMaster Universities OA Index (WOMAC ${ }^{\text {TM }}$ Index) [8, 9]. This is a self-administered and patient-reported assessment OA of knee status.

2. The index is tri-dimensional covering pain (item 1-5), stiffness (item 6-7), and physical functional (item 8-24).
3. The patients assess the severity of pain during walking, climbing stairs, during sleep, sitting, and standing. Patient's assessment of stiffness in the morning and for the rest of the day.

4. The physical function subscales assess difficulty in performing the following functions: descending stairs; ascending stairs; getting out of the chair; remaining in standing position; bending; walking on a flat surface; in/out of the car; shopping; stockings on; getting out of bed; stockings off; lying in bed, in/ out bath; sitting; toileting; heavy domestic duties; and light domestic duties.

5. The response was graded by 5-point Likert intensity scale (none, mild, moderate, severe, or extreme) with a final score range from 0 to 96 .

6 . The higher score reflects worsening of pain and a poorer function.

\section{Ethical approval protocol}

All authors hereby declare that all experiments have been examined and approved by the appropriate Ethics Committee from Aljamhoori Teaching Hospital, Iraqi Ministry of Health (code:2020111) and Faculty of Medicine/Mosul University (code: 779RHEU01) and have therefore been performed under the ethical standards laid down in the 1964 Declaration of Helsinki.

\section{Participants informed consent}

All authors declare that written informed consent was obtained from the patient (or other approved parties) for publication of this article.

\section{Statistical analysis}

All the data has been entered and processed using statistical package SPSS ver. 24 (Chicago Inc., III). Different descriptive statistical testes and graphic techniques (mean, SD, and percentile) were used to summarize and tabulate the data. The significance of changes in the WOMAC score from baseline to follow-up was assessed using Wilcoxon signed-rank test. The changes are reported as mean with 95\% confidence intervals $(\mathrm{Cl})$ of difference. A p-value $\leq$ 0.05 was considered statistically significant.

\section{RESULTS}

\section{Demographic characteristics of patients and $O A$}

17 patients with OA were eligible for the current study and agreed to participate. 10 patients had stage III and 7 had stage IV. Of them, 11 patients with unilateral knee $O A$ and 6 patients with bilateral knee OA were included. The age of patients ranged from 45 to 81 years, with mean \pm SD of $61.35 \pm 8.74$ 
years. The BMI of the patients ranged from 24.5 $8.6 \mathrm{~kg} / \mathrm{m}^{2}$ with a mean \pm SD of $31.79 \pm 4.27 \mathrm{~kg} / \mathrm{m}^{2}$. The duration of OA ranged from $1-5$ years with a mean \pm SD of $5.17 \pm 3.76$ years. The characteristics of the studied sample are shown in Table 1.

\section{Open clinical trial results}

The changes in individual subscales and total scores of WOMAC are shown in Table 2 and Figure 1. The results revealed that the pain subscale was reduced by an average -3.88 , stiffness subscale was reduced by an average -1.29 , physical function subscales were reduced by an average of 9.29 , and the total score was reduced by an average of -14.47 . Because of the uneven distribution of the number of items among subscales, the pre- and post-treatment differences were divided by their corresponding number of items to yield mean changes. The changes in descending frequencies were as follows: the pain subscales, stiffness subscale, total score, and lastly, in the subscale of physical function. As statistical significance is not everything, further analysis was performed using Cohen's d formula to estimate effect size. It was calculated by a measurement of the distance between the pre- and post-treatment means divided by their pooled standard deviations [10]. The measured effect size in descending pattern was as follows: stiffness subscale (1.49); pain subscale (1.23); total WOMAC score (1.20); and lastly physical function subscale (1.11). In summary, the effect size of the combination of PRP and HA intra-articular injection is classified as large effect size (i.e., > 0.8) in improving the outcome of knee OA.

Table 1. Demographic and clinical characteristics of the studied sample of Osteoarthritis patients

\begin{tabular}{|l|c|}
\hline Parameters & \\
\hline Age in years, mean (SD) & $61.35 \pm 8.74$ \\
\hline Duration of OA in years, mean (SD) & $5.17 \pm 3.76$ \\
\hline BMl in kg/m ${ }^{2}$ mean (SD) & $31.79 \pm 4.27$ \\
\hline Site of OA, No. (\%) & \\
Right & $8(47.1 \%)$ \\
Left & $3(17.6 \%)$ \\
Both & $6(35.3 \%)$ \\
\hline Stage of OA, No. (\%) & $10(58.8 \%)$ \\
III & $7(41.2 \%)$ \\
IV & \\
\hline
\end{tabular}

Table 2. Comparison of changes of WOMAC subscales and total scores after 3 intra-articular injections of a combination of PRP and HA for Knee OA

\begin{tabular}{|l|c|c|c|}
\hline WoMAC subscales & $\begin{array}{c}\text { Pre-treatment } \\
\text { Mean } \pm \text { SD }\end{array}$ & $\begin{array}{c}\text { Post-treatment } \\
\text { Mean } \pm \text { SD }\end{array}$ & $\begin{array}{c}\text { 95\% Cl of difference } \\
\text { Min-Max }\end{array}$ \\
\hline Pain & $11.23 \pm 3.40$ & $7.35 \pm 2.87$ & $-3.18-4.58$ \\
\hline Stiffness & $4.88 \pm 1.26$ & $3.58 \pm 1.22$ & $-0.85-1.73$ \\
\hline Physical function & $36.88 \pm 10.11$ & $27.58 \pm 6.16$ & $-6.70-11.88$ \\
\hline Total & $53.00 \pm 14.29$ & $38.52 \pm 9.31$ & $-11.20-17.73$ \\
\hline
\end{tabular}

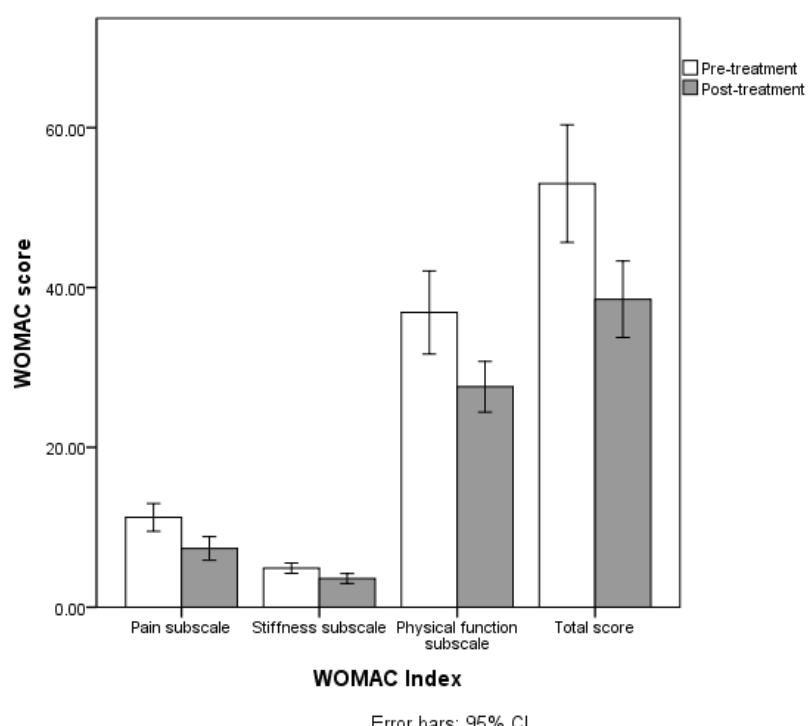

Error bars: $95 \% \mathrm{Cl}$
Fig. 1. The pre- and post-treatment changes in pain, stiffness, physical function and total score of WOMAC index after three sessions of intra-articular injection of a combination of PRP and HA 


\section{Discussion}

Because of the lack of treatment guidelines for $\mathrm{OA}$, the researchers revised published trials aiming to determine an empirically tested proper schedule (the number of injections and time length between them) for the current study. Three studies were found to be relevant. First, the superiority of multiple intra-articular injections over a single injection by Kavadar et al. [10] was considered. Second, Görmeli et al. [7] found that 3 PRP injections represent the best frequency of intra-articular injection in achieving the best clinical results. Regarding interval between injections, Montanez-Heredia et al. adopted two weeks [11], Li et al. [12] used a three-week interval, while Duymus et al. [13] adopted a monthly injection with best results. Based on these studies, the regimen adopted in this study consists of three injections with a month interval between injections. In vivo studies have elaborated on the effectiveness of PRP and HA in treating musculoskeletal disorders [14]. Sundman. et al. infer from their co-culture model of synovial cells and chondrocytes that both medications treat OA via different mechanisms (HA mimics synovial fluid and helps in replenishing ill-synovial fluid while PRP delivers factors that modulate angiogenesis, inflammation, and cell anabolism) [15]. These basic studies revealed that the action of PRP and HA can be synergistically associated to regenerate cartilage without changing characteristics of both medications [16]. Based on these results, the physician may blend both treatments and benefit from their dissimilar biological mechanisms. These regimens are expected to become a widely used clinical practice in repairing knee OA [16].

The results of the current study proved that repetitive injections of PRP and HA alleviate symptoms in OA patients. The outcomes of the current study were compared with pooled outcomes of 15 randomized clinical trials $(n=1314)$ used PRP or HA in Knee OA. The studies using WOMAC in assessment of outcomes of therapy were retrieved from the electronic database up to April 2018. The calculated effect size of the combination of PRP and HA used in the current study on pain subscale was 1.23 , which was a higher figure reported after $\mathrm{HA}(0.94)$ but was lower than that reported after PRP (1.5). The calculated effect size of the combination of PRP and HA used in the current study on stiffness subscale was 1.49 , which was far higher than the figure reported after HA (0.35) and after PRP (0.89). The calculated effect size of the combination of PRP and HA used in the current study on the physical function subscale was 1.11 , which was a higher figure reported after HA (0.55) and after PRP (0.83). Overall, the calculated effect size of the combination of PRP and HA used in the current study on the total score of the WOMAC index was 1.20 , which was a higher figure reported after HA (0.66) and after PRP (0.97).

The above-proposed treatment regimens of a combination of intra-articular PRP and HA injections were similar to the results reported by Georgiev and Stoilov, in which they found a statistically significant alleviation of pain and improvement of disease activity, and more than half of the participants responded positively to therapy in both groups of the study after one year [17]. Ruther more, Wu et al. based on their study evidence found that the PRP appears to be better for pain relief and self-reported functional improvement than HA, but they did not use combination modalities [18].

\section{Conclusion}

A combination of PRP and HA has a large synergistic effect size in improving the outcome of moderate and severe OA of the knee. Most of the patients with knee OA presented with advanced stages, and old age groups. Pain subscale, stiffness subscale, and physical function subscales were reduced. The smallest effect size was found after HA intra-articular injection. The PRP produced an intermediate effect size. The effect size of the combination of PRP and $\mathrm{HA}$ intra-articular injection is classified as a large effect size in improving the outcome of knee OA.

Funding source

No funding sources.

Declaration of Competing Interest

None.

\section{References}

1. Zhang Y, Jordan JM. Epidemiology of Osteoarthritis. Clin Geriatr Med. 2010; 26(3): 355-369.

2. Kubcek M, Florian Z. Stress-strain analysis of knee joint. Engineering Mechanics 2009; 16 (5): 315-322

3. Buckwalter JA, Brown TD. Joint injury, repair, and remodeling: roles in post-traumatic osteoarthritis. Clin Orthop Relat Res, 2004;423: 7-16.

4. Laudy AB, Bakker EW, Rekers M, et al. Efficacy of platelet-rich plasma injections in osteoarthritis of the knee: A systematic review and meta-analysis. $\mathrm{Br} \mathrm{J}$ Sports Med. 2015;49:657-672.

5. Altman RD, Manjoo A, Fierlinger A, et al. The mechanism of action for hyaluronic acid treatment in the osteoarthritic knee: A systematic review. BMC Musculoskeletal Disord. 2015;16:321 
6. Ahadi T, Abtahi M. Platelet-rich plasma versus hyaluronic acid. Arthroscopy. 2012;28:1585.

7. Görmeli G, Görmeli CA, Ataoglu B, et al. Multiple PRP injections are more effective than single injections and hyaluronic acid in knees with early osteoarthritis: A randomized, double-blind, placebo-controlled trial. Knee Surg Sports Traumatol Arthrosc. 2017;25:958-965.

8. Faik A, Benbouazza K, Amine B, et al. Translation and validation of Moroccan Western Ontario and McMaster Universities (WOMAC) osteoarthritis index in knee osteoarthritis. Rheumatol Int. 2008 May; 28(7):677-83.

9. Durlak, J. How to Select, Calculate, and Interpret Effect Sizes. Journal of Pediatric Psychology. 2009: 34(9):917-28.

10. Kavadar G, Demircioglu DT, Celik MY, et al. Effectiveness of platelet-rich plasma in the treatment of moderate knee osteoarthritis: a randomized prospective study. J Phys Ther Sci. 2015;27(12):3863-7.

11. Montanez-Heredia E, Irizar S, Huertas PJ et al. Intra-articular injections of platelet-rich plasma versus hyaluronic acid in the treatment of osteoarthritic knee pain: A randomized clinical trial in the context of the Spanish National Health Care System. Int J Mol Sci 2016;17(7):1064-1077.

12. Li M, Zhang C, Ai Z, et al. Therapeutic effectiveness of intra-knee-articular injection of platelet-rich plasma on knee articular cartilage degeneration [in Chinese] Zhongguo Xiu Fu Chong Jian Wai Ke Za Zhi 2011;25:1192-6.

13. Duymus TM, Mutlu S, Dernek B, et al. Choice of intra-articular injection in the treatment of knee osteoarthritis: Platelet-rich plasma, hyaluronic acid, or ozone options. Knee Surg Sports Traumatol Arthrosc 2017; 25 (2):485-92.
14. Han $\mathrm{Y}$, Huang $\mathrm{H}$, Pan J, et al. Meta-analysis Comparing Platelet-Rich Plasma vs Hyaluronic Acid Injection in Patients with Knee Osteoarthritis. Pain Medicine 2019;20(4):661-87.

15. Sundman EA, Cole BJ, Karas V, et al. The Anti-inflammatory and Matrix Restorative Mechanisms of Platelet-Rich Plasma in Osteoarthritis. Am J Sports Med. 2014;42(1) 35-41.

16. Chen WH, Lo WC, Hsu WC, et al. Synergistic anabolic actions of hyaluronic acid and platelet-rich plasma on cartilage regeneration in osteoarthritis therapy. Biomaterials. 2014;35(36) 9599-9607.

17. Georgiev T, Stoilov R. High molecular-weight hyaluronan or rich plasma platelet for osteoarthritis knee - real-world experience from specific treatment regimens. Osteoarthritis and Cartilage. 2020;28(1): S497-S498.

18. Wu Q, Luo X, Xiong Y, et al. Platelet-rich plasma versus hyaluronic acid in knee osteoarthritis: A meta-analysis with the consistent ratio of injection. J Orthop Surg (Hong Kong). 2020;28(1):2309499019887660.

Submitted: 06.02.2017

Correspondence address:

Ramzi Ramzi

Department of cardiothoracic surgery, Aljamhoori Teaching

Hospital, Mosul Health Directorate

Ministry of Health/Environment, Mosul, Iraq

E-mail: Ali2014201466@yahoo.com

009627703272132

ZC: 64001 